[Agr. Biol. Chem., Vol. 31, No. 11, p. 1270 1275, 1967]

\title{
The Nutritional Value of the Individual Nonessential Amino Acid as the Nitrogen Source in the Chick Nutrition
}

\author{
By Michihiro Sugahara and Shujiro AriYoshi \\ Research Laboratory of Amino Feed Co., Inc., Central Research Laboratories, \\ Ajinomoto Co., Inc., Totsukaku, Yokohama, Japan \\ Received May 25, 1967
}

\begin{abstract}
The nutritional values of nonessential amino acids as the nitrogen source in the crystalline amino acid diet for the chick growth were examined. The nitrogen of the nonessential amino acids in the basal diet for chick was substituted for a nonessential amino acid to be tested on the nitrogen base. The experimental methods were the same as in the evaluation of the nutritional value of $\mathrm{D}$-amino acids previously reported. Nonessential amino acids were classified into four groups.

A. Very useful nitrogen source: Glutamic acid, Aspartic acid

B. Useful nitrogen source: Alanine, Diammonium citrate

C. Insufficient nitrogen source: Glycine, Proline

D. Harmful for chick growth: Serine

At the end of experiment chicks were killed and the concentration of free amino acids in the serum were measured. The concentration of glycine and serine in the serum increased when glycine was tested, but that of serine in the serum only increased when serine was tested. This result suggested the pathway from glycine to serine was fast and the opposite one was very slow.
\end{abstract}

Since Almquist and Grau" performed the growth test of chick by the crystalline amino acid diet as a sole source of nitrogen, many experiments using purified amino acid diet were carried out. The compositions of the amino acid diet in each experiment was slightly different one another.

The developement of the crystalline amino acid diet has mainly based on the improvement of the composition of the essential amino acid and on the nitrogen content in the diet. At early time all nonessential amino acids were used as the component, but Klain et al. ${ }^{2 \prime}$ simplified the composition of the nonessential amino acid. They removed alanine, serine, aspartic acid and proline from the nonessential

1) H. J. Almquist and C. R. Grau, J. Nutrition, 28, 325 (1944).

2) G. J. Klain, H. M. Scott and B. Connor Johnson, Poultry Sci., 38, 489 (1959). amino acid mixture. The nitrogen of the removed nonessential amino acids was substituted for the nitrogen of glutamic acid on the nitrogen base. They included cystine and tyrosine in the group of the essential amino acid. Soon after Greene et al. $^{3 !}$ had found the essentiality of L-proline for the chick growth on the crystalline amino acid diet, the same observation was taken in our laboratory. ${ }^{4 !}$

Recently the importance of dispensable amino acid for normal growth of chick was considered, ${ }^{51}$ but the nutritional value of each nonessential amino acid as the nitrogen source in the protein metabolism was not investigated.

3) D. E. Greene, H. M. Scott and B. C. Johnson: Poultry Sci., 41, 116 (1962)

4) M. Sugahara and S. Ariyoshi, This Journal, 31: 106 (1967).

5) W. P. Stucki and A. E. Harper, J. Nutrition 74, 377 (1961). 
Table I. Composition of Basal and Test Diet $(\%)$

Ingredient

\section{Corn starch}

Soybean oil

Mineral** mixture

Vitamine*** mixture

Vitamine*** $\mathrm{AD}_{3}$

Cellulose powder

Agar-agar

$\mathrm{NaHCO}_{3}$

Anti-acid:

Amino acid玤 mixture

L-Arg- $\mathrm{HCl}$

L-His-HCl

L-Lys-HCl

L-Tyr

L-Try

L-Phe

L-Cys

L-Met

L-Ser

L-Thr

L-Leu

L-ILeu

$\mathrm{L}-\mathrm{Val}$

L-Glu

L-Asp

L-Ala

L-Pro

Gly

D.A.C.*

* Diammonium citrate

** G. J. Klain et al. $($ (960)6

*** M. Yoshida et al. $(1963)^{7}$ )

- Consists of equal parts of alminium hydroxide and magnesium trisilicate.

* Composition of amino acids mixture is almost in the same proportion as found in the whole egg protein. ${ }^{8}$ Protein Content $(\mathrm{N} \times 6.25)=21.1 \%$.

The experiment reported here were undertaken to clear the specific character of each nonessential amino acid in the chick nutrition as the nitrogen source. In this experiment glycine was included in the group of nonessential amino acid. Although the essentiality of $\mathrm{L}$-proline in the crystalline amino acid diet

6) G. J. Klain, H. M. Scott and B. C. Johnson, Poultry Sci., 39, 39 (1960).

8) M. Yoshida, H. Hoshii and H. Morimoto, $J$. Agr. Chem. Soc. Japan, 37, 187 (1963).

9) R.J. Block, "Amino Acid Handbook", Charles C. Thomas Publisher, 1956 , p. 341 . is apparent, ${ }^{3,4,9)}$ the nutritional value of $\mathbf{L}^{-}$ proline as the nitrogen source was also investigated in order to compare with that of glycine which has come into question on its essentiality in chick nutrition."

\section{EXPERIMENTAL}

The growth of chick in this experiment was tested by the same method as previously reported ${ }^{10}$ ) (except

9) D. A. Benton, H. E. Spivey, A. E. Harper and C. A. Elvehjem, Arch. Biochem. Biophys., 57, 262 (1955).

10) M. Sugahara, T. Morimoto, T. Kobayashi and S. Ariyoshi, This Journal, 31, 77 (1967). 
Table II. Feed Consumption, Growth Rate, Feed Efficiency, Nitrogen Retention, Nitrogen EFficiency* and Protein EFficiency Ratio on the Basal

DIET AND THE TEST DIETS

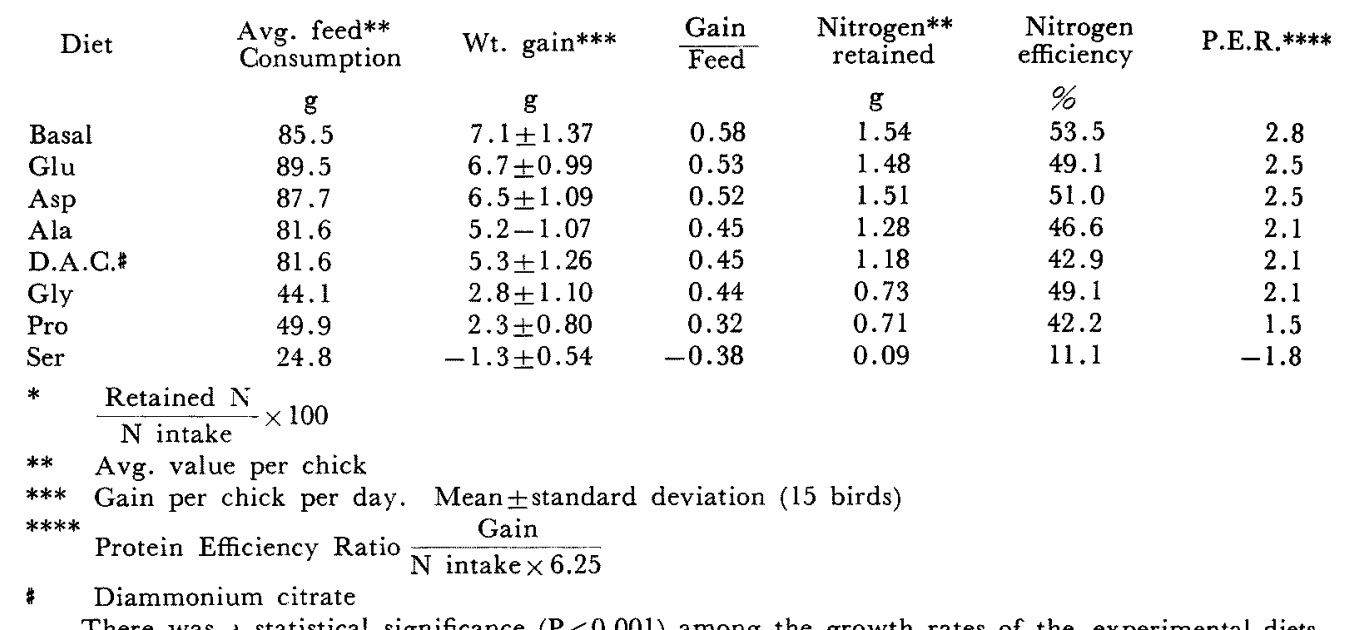

No statistical significance was found among the growth rates of the basal, glutamic acid and aspartic acid diet. There was a statistical significance $(\mathrm{P}<0.05)$ among the growth rates of the basal, alanine and diammonium citrate diet.

the number of test birds). Nonessential amino acids* except a nonessential amino acid to be tested were rernoved from the basal diet and the nitrogen of the removed amino acids was substituted for the nitrogen of an amino acid to be tested on the nitrogen base. Cystine, tyrosine and proline were regarded as the essential component. When the amount of the amino acid mixture changed, the amount of starch was regulated. The composition of the amino adid of the test diet was shown in Table I. Diammonium citrate was chosen as an unnatural nitrogen donor.

At the end of the experiment chicks were killed by cutting the carotid artery. The blood was received in a beaker containing sodium oxalate. The serum was collected by centrifugation $(3,000$ r.p.m. $5 \mathrm{mi}$ nutes). Serum-protein was preciptated by adding trichloroacetic acid to make $10 \%$ (W/V). Supernatant was collected by centrifugation and trichloroacetic acid was removed by ether extraction. After the extraction of trichloroacetic acid, the serum was dried up in vaccuo. The concentration of the amino acid was determined by the automatic amino acid analyzer (Hitachi KLA-2).

* Glu, Asp, Ala, Gily, Ser.

\section{RESULTS}

Growth curves of chicks was shown in Fig. 1. Feed consumption, growth rate, feed ef-



Fig. 1. Growth Curves of Chicks Fed on Basal and Test Diet. 
ficiency, nitrogen efficiency and protein efciency ratio were shown in Table II.

L-Glutamic acid. The chicks fed on the Lglutamic acid diet showed less growth than those fed on the basal diet, though there was no statistical significance between both group. Feed efficiency, nitrogen efficiency and protein efficiency ratio of chicks fed on the L-glutamic acid diet were all a little inferior to those of chicks fed on the basal diet.

L-Aspartic acid. The chicks fed on the Laspartic acid diet grew almost at the same rate as the chicks fed on the L-glutamic acid diet. Therefore the nutritional value of L-glutamic acid or L-aspartic acid as the nitrogen source for the chick growth may be slightly inferior to that of the mixture of all nonessential amino acids based on the whole egg pattern.

L-Alanine. The chicks fed on the L-alanine diet grew appreciably slower than the chicks fed on the basal diet. The nutritional value of L-alanine as the nitrogen source is clearly inferior to that of L-glutamic acid or L-aspartic acid.

Diammonium citrate. The chicks fed on the diammonium citrate diet showed the same growth rate in comparison with that of the chicks fed on the L-alanine diet. It is interesting that diammonium citrate is a useful nitrogen source though it is an unnatural substance.

Glycine. Glycine may be considerably inferior nitrogen source compared with L-glutamic acid or L-aspartic acid. Growth rate and feed consumption of chicks fed on glycine diet were almost one half of those of chicks fed on the L-glutamic acid diet.

L-Proline. The chicks fed on the L-proline diet showed less growth rate, feed conversion and protein efficiency ratio than those of the chicks fed on the glycine diet. From third day to seventh day growth retardation of chicks fed on the proline diet was evident, but the chicks on the glycine diet group grew at the constant rate during the experimental

Table III. The Concentration of Free amino acid in the Serum Fed on the Basal Diet and The Test Diets $(\gamma / \mathrm{ml})$

\begin{tabular}{|c|c|c|c|c|c|c|c|c|}
\hline \multirow{2}{*}{ Amino acid } & \multicolumn{8}{|c|}{ Diet } \\
\hline & $\overparen{\text { Basal }}$ & Glu & Asp & Ala & D.A.C.\# & Gly & Pro & $\widehat{\operatorname{Ser}}$ \\
\hline Try & 13.0 & 11.2 & $*$ & 6.8 & 10.8 & & $*$ & $*$ \\
\hline Lys & 232.0 & 303.2 & 243.7 & 268.0 & 313.4 & 172.8 & 193.6 & 152.2 \\
\hline $\mathrm{His}$ & 19.5 & 16.9 & 13.9 & 27.4 & 10.8 & 21.3 & 27.8 & 18.5 \\
\hline Arg & 45.5 & 28.1 & 13.9 & 68.5 & 53.8 & 85.1 & 64.8 & 74.1 \\
\hline Tau & $*$ & $*$ & 13.9 & 34.2 & 32.3 & 56.7 & 37.0 & 55.6 \\
\hline Asp & 30.0 & 16.9 & 41.7 & 20.5 & 41.8 & 14.2 & 18.5 & 18.5 \\
\hline Thr & 402.6 & 269.7 & 125.1 & 404.1 & 655.9 & 340.4 & 324.0 & 240.7 \\
\hline Ser & 230.0 & 72.7 & 34.6 & 92.8 & 80.4 & 562.7 & 113.9 & 853.0 \\
\hline Glu & 45.5 & 50.7 & 13.1 & 48.0 & 32.3 & 42.6 & 55.5 & 37.0 \\
\hline Pro & 58.5 & 67.4 & 27.8 & 95.9 & 69.9 & 70.9 & 201.9 & 74.1 \\
\hline Gly & 71.4 & 22.5 & 13.9 & 34.2 & 32.3 & 631.2 & 37.0 & 92.6 \\
\hline Ala & 142.8 & 140.5 & 41.7 & 315.1 & 64.5 & 70.9 & 240.7 & 55.6 \\
\hline Cys & $*$ & & $*$ & $*$ & * & $*$ & * & $*$ \\
\hline Val & 58.5 & 67.4 & 27.8 & 116.4 & 91.4 & 70.9 & 92.6 & 74.1 \\
\hline Met & 13.0 & 28.1 & 13.9 & 48.0 & 74.1 & 28.4 & 27.8 & 18.5 \\
\hline Ileu & 32.5 & 39.3 & 13.9 & 48.0 & 74.1 & 35.5 & 46.3 & 37.0 \\
\hline Leu & 39.0 & 39.3 & 13.9 & 68.5 & 59.1 & 42.6 & 55.5 & 37.0 \\
\hline Tyr & 78.0 & 61.8 & 27.8 & 96.0 & 139.8 & 85.1 & 64.8 & 37.0 \\
\hline Phe & 30.0 & 22.5 & 13.9 & 41.1 & 74.1 & 28.4 & 27.8 & 18.5 \\
\hline
\end{tabular}

* It was not determined by the disturbance of unknown substance(s).

* Diammonium citrate. 
period. Proline may be more inefficient nitrogen source than glycine.

L-Serine. The chicks fed on the L-serine diet decreased their body weight in such pattern as observed in the group deficient in an essential amino acid. ${ }^{10}$ The excess of serine in the diet was harmful to the chick growth.

From the results shown above, nonessential amino acids can be classified into four groups as the nitrogen source in the crystalline amino acid diet for the chick growth.

A. Very useful nitrogen source Glutamic acid, Aspartic acid

B. Useful nitrogen source Alanine, Diammonium citrate

C. Non-suitable nitrogen source Glycine, Proline

D. Harmful for chick growth Serine

The concentration of the free amino acids in the serum of chicks fed on test diet was shown in Table III. When the mixture of all nonessential amino acids was substituted for a nonessential amino acid to be tested, the concentration of the free amino acid under testing increased in comparison with that of the chicks fed on the basal diet except glutamic acid and aspartic acid. The concentration of alanine increased as much as 2.2 times than that of the control group. The increased ratios of other amino acids were as follows. Glycine 8.8 times; proline 3.5 times; serine 3.7 times.

In the glycine diet group the content of serine in the serum was similarly increased as that of glycine, but in the serine diet group the increase of the glycine content in the serum was not clear in spite of apparent increases of the serine content.

\section{DISCUSSION}

Recently L-glutamic acid is used as a sole source of nonessential amino acid in the crystalline amino acid diet. The findings obtained in this experiment show that the efficiency of the utilization of the nitrogen of glutamic acid for the protein metabolism is slightly inferior to that of the mixture of all nonessential amino acids based on the whole egg pattern. The optimum pattern of nonessential amino acid for the maximum chick growth must exist. However there actually may be some optimum patterns, because the change of dietary conditions such as essential amino acid pattern, crude protein content or protein-calorie ratio has the influence on the pattern of nonessential amino acid.

It is clear that the nutritional value of $\mathrm{L}^{-}$ alanine as the nitrogen source is inferior than L-glutamic acid or L-aspartic acid. Glutamic acid, aspartic acid and alanine are considered as the main nitrogen donors in protein metabolism. They relate each other through tricarboxylic acid cycle via $\alpha$-ketoglutaric acid, oxaloacetic acid and pyruvic acid, respectively. Alanine and aspartic acid are not connected with each other by transamination, but alanine and glutamic acid are connected with each other by glutamic-pyruvic transaminase. Glutamic acid and aspartic acid are connected with each other by glutamic-oxaloacetic transaminase.

The results obtained in this experiment might show that the pathway (alanine $+\alpha-$ ketoglutaric acid $\rightarrow$ glutamic acid + pyruvic acid) is slow compared with that of opposite reaction. On the other hand, the reaction of glutamic-oxaloacetic transamination is fast for both directions.

It is interesting that unnatural substance (diammonium citrate) has the same efficiency as the nitrogen source compared with alanine. Rose et al. ${ }^{11}$ fed weanling rats on a diet in which virtually all of the nitrogen was in the form of the ten essential amino acids, each at the lowest level. They added L-glutamic acid, glycine, diammonium citrate and urea as the nonessential nitrogen supplement. They found diammonium citrate and glutamic acid

11) W. C. Rose, L. C. Smith, M. Womack and M. Shane, J. Biol. Chem., 307, 181 (1949). 
were the most effective, glycine was intermediate, and urea was the least active. They concluded these variations in potency were doubtlessly to be accounted for by differences in the facility with which the compounds in question may serve as sources of nitrogen for amination or transamination reactions.

Serine is usually considered a main precursor of glycine in protein metabolism. If the concentration of free amino acid in the serum reveals the dynamic aspect of protein metabolism, the result in this experiment shows the conversion from glycine to serine is considerably fast, but the opposite conversion is very slow. It is desirable in chick nutrition to examine the validity of the general conception that serine is a main precursor of glycine, though metabolic difference between endogenous synthesized and exogenous added serine may exist and the condition of chicks fed on both experimental diets was not always normal.

When glutamic acid or aspartic acid was tested, the concentration of free glutamic acid or aspartic acid in the serum was not increased in comparison with that in the case of basal diet. This result shows that large amount of glutamic acid or aspartic acid is utilized smoothly as the nitrogen source.

However in the cases of alanine, glycine, proline and serine, the concentration of an amino acid under testing always increased. These results show the pathways of these amino acids for the utilization as the nitrogen source are insufficient, then some parts of absorbed amino acid may overflow into blood stream. Increased free amino acid in the serum may give some effects for physiological conditions of chick. As the over-all result, chick decreases nitrogen retention and growth rate.

Acknow ledgement. We wish to thank Miss Kiiko Tenmyō for many help in the performance of this experiment. 